\title{
Chemical recycling of PET by alkaline hydrolysis in the presence of quaternary phosphonium and ammonium salts as phase transfer catalysts
}

\author{
R. López-Fonseca, M. P. González-Marcos, \\ J. R. González-Velasco \& J. I. Gutiérrez-Ortiz \\ Chemical Technologies for Environmental Sustainability Group, \\ Department of Chemical Engineering, \\ Faculty of Science and Technology, Universidad del País Vasco/EHU, \\ Bilbao, Spain
}

\begin{abstract}
Chemical or tertiary recycling of waste polymers including PET, poly(ethylene terephthalate), is the only method according to the principles of Sustainable Development, since it leads to the formation of the raw materials (monomers) from which the polymer is made of. This work has been focused on the determination of the kinetics of the depolymerisation of PET by means of alkaline hydrolysis and on the identification of the catalytic behaviour, if any, of a series of phosphonium and ammonium salts as phase transfer catalysts (9 salts with varying alkyl groups, central cation ( $\mathrm{N}$ or $\mathrm{P}$ ) and anion $\left(\mathrm{Cl}^{-}, \mathrm{Br}^{-}, \mathrm{I}^{-}, \mathrm{OH}^{-}\right)$).

Among the catalysts tested tributylhexadecylphosphonium bromide (3Bu6DPB) is found to be the most effective catalyst. Complete conversion of PET with considerably low catalyst concentration and temperature can be achieved. The selected phase transfer catalyst fulfils the requirements of having enough character in order to be lipophilic, while small enough in order to avoid steric hindrance. The time for complete hydrolysis ( $>90 \%$ conversion) of solid PET under alkaline conditions with $3 \mathrm{Bu} 6 \mathrm{DPB}$ at $80^{\circ} \mathrm{C}$ is 1.5 hours and 10 hours without 3Bu6DPB.
\end{abstract}

Keywords: chemical recycling, PET, alkaline hydrolysis, kinetics, phase transfer catalysts. 


\section{Introduction}

Poly(ethylene terephthalate) (PET) is a semicrystalline polyester showing excellent thermal and mechanical properties. Although its main application was predominately textile industry, substantial quantities of this material are consumed in the manufacture of video and audio tape, food packaging and especially of soft-drink bottles. It should be pointed out that PET does not create a direct hazard to the environment, but owing to its substantial fraction by volume in the waste stream and its high resistance to both atmospheric and biological agents, it is seen as a noxious material.

PET recycling represents one of the most successful and widespread examples of polymer recycling. Petcore recently announced that European postconsumer PET collection rates reached 944,000 tonnes in 2006, an $18.5 \%$ increase over the previous year [1]. The increase in PET collection continues, by far, to exceed growth in consumption with $38.6 \%$ of all PET bottles being collected for recycle. Collection continued to grow steadily in most European countries with the highest increase in rates where national legislation is changing to allow single trip bottles to replace refillable containers.

The recycling of waste polymers including PET can be carried out in many ways [2]. However, the only method acceptable according the principles of Sustainable Development is the so-called tertiary or chemical recycling, since it results in the yield of the corresponding monomers. The chemical recycling of PET can be conducted by means of the following processes: (i) glycolysis, (ii) methanolysis, (iii) hydrolysis and (iv) aminolysis or ammonolysis. All these methods have been recently reviewed by Paszun and Spychaj [3], Karayannidis and Achilias [4] and Lorenzetti et al [5]. Nowadays there is a growing interest in hydrolysis for the chemical recycling of PET, since it is the only method that leads to terephthalic acid (TPA) and ethylene glycol (EG) as reaction products, i.e., the monomers from which PET is formed. This is associated with the trend in new factories for PET synthesis to produce it directly from TPA and EG, thus replacing dimethyl terephthalate (the traditional monomer) from the technological process. Hydrolysis can be carried out under (a) alkaline, (b) acid and (c) neutral conditions.

In the present investigation the interest has been focused, on one hand, on the kinetic modelling of the alkaline hydrolysis, and, on the other hand, on a screening study of different quaternary phosphonium or ammonium salts as efficient phase transfer catalysts (PTCs) for this process.

\section{Experimental part}

Pure PET flakes (Aldrich) were used in the experiments of depolymerisation in a sodium hydroxide solution. Flakes were previously cut with a cryogenic rotatory cutter (Restch ZM 2000). Reaction experiments were carried out in a $300 \mathrm{ml}$ capacity Parr pressure reactor equipped with a motor for stirring to ensure proper mixing and an electric heating mantle in order to reach the reaction temperature. A nitrogen purge was used to create an inert atmosphere inside the vessel. PET 
flakes (10 g), sodium hydroxide (10 g), PTC (1.4, 2.7 or $5.5 \mathrm{~g})$ and water (150 g) were charged into the reactor at room temperature and then heated to the selected temperature $\left(60-100{ }^{\circ} \mathrm{C}\right)$ for conducting the hydrolytic runs. The mixture was allowed to react for 1.5-4 hours. After the required time interval for reaction was reached, the vessel was quickly removed from the heating mantle and immersed in an ice bath. The temperature of the vessel was quenched to room conditions so as to interrupt the progress of hydrolysis. The residual flakes and the alkaline solution containing the PTC were separated by filtration (Schleider \& Scheull GF6 binder glass microfiber filter) under vacuum. The flakes were thoroughly washed with deionised water, dried at $110^{\circ} \mathrm{C}$ for one hour, and weighed. The melting thermogram of the residual PET was investigated by differential scanning calorimetry (Mettler Toledo DSC 822e) at a heating rate of $10^{\circ} \mathrm{C} \mathrm{min}^{-1}$. Also the size of partially converted PET flakes was measured by laser scattering (Malvern Mastersizer X).

After filtration disodium terephthalate, ethylene glycol, and the PTC were present in the liquid phase. Excess hydrogen chloride was added into the filtrate in order to neutralise the sodium hydroxide and provoke the precipitation of the terephthalic acid (TPA). This solid product was filtered under vacuum, further washed with deionised water, dried at $110^{\circ} \mathrm{C}$ and weighed. The remaining liquid essentially composed of ethylene glycol and water was directly used for analysis.

The carboxylic acid concentration in the solid products was determined by potential titration [6]. A total of $25 \mathrm{ml}$ of deuterated dimethyl sulfoxide was used as the solvent, in which $0.1 \mathrm{~g}$ of the solid product was dissolved. The solution was titrated with a $0.1 \mathrm{~N}$ potassium hydroxide/ethanol solution at room temperature. The potential of the solution versus the amount of the titrant recorded was recorded, and the plot was then used to determine the phenolphthalein end point of titration. Also the purity of the separated TPA was determined by ${ }^{1} \mathrm{H}-\mathrm{NMR}$ (Rutina Broker AC-250).

Table 1: Quaternary phosphonium or ammonium salts used as PTCs in the alkaline depolymerisation of PET.

\begin{tabular}{|c|c|}
\hline Acronym & Quaternary salt \\
\hline 4BuAB & Tetrabutylammonium bromide \\
\hline 4OAB & Tetraoctylammonium bromide \\
\hline 4BuACl & Tetrabutylammonium chloride \\
\hline 4MPB & Tetramethylphosphonium bromide \\
\hline 4BuPB & Tetrabutylphosphonium bromide \\
\hline 4OPB & Tetraoctylphosphonium bromide \\
\hline 3Bu6DPB & Tributylhexadecylphosphonium bromide \\
\hline 4BuPCl & Tetrabutylphosphonium chloride \\
\hline 4BuPH & Tetrabutylphosphonium hydroxide \\
\hline
\end{tabular}

The concentrations of the organic products in the remaining liquid were quantitatively analysed by gas chromatography in order to ascertain the presence of products derived from secondary reactions (for example, diethylene glycol). 
Methanol was used as an internal standard. The mixed solution was injected into a gas chromatograph (Agilent Technologies 6890N Network GC System) and separated by a $30 \mathrm{~m}$ capillary column (DB-624) with an internal diameter of 3 $\mu \mathrm{m}$. The components were carried by a helium flow and detected by a flame ionization detector.

With the aim of decreasing the operating temperatures and/or the reaction time required to achieve high conversions a wide number of PTCs, namely quaternary phosphonium or ammonium salts (9 PTCs with varying alkyl groups, central cation $(\mathrm{N}$ or $\mathrm{P})$ and anion $\left(\mathrm{Cl}^{-}, \mathrm{Br}^{-}, \mathrm{I}^{-}, \mathrm{OH}^{-}\right)$), were examined for their activity in the depolymerisation process. The salts used in this study are listed in table 1 . All of them were supplied by the Aldrich Chemical Company.

\section{Results and discussion}

\subsection{Non-catalysed alkaline hydrolysis of PET}

PET flakes were hydrolysed in sodium hydroxide to yield the disodium salt and ethylene glycol according to the following chemical reaction:

$$
P E T(s)+2 \mathrm{NaOH}(l) \rightarrow \mathrm{Na}_{2}-\mathrm{TPA}(l)+E G(l)
$$

PET conversion was calculated using the following equation:

$$
\text { PET conversion }(\%)=\frac{W_{P E T, 0}-W_{P E T, t}}{W_{P E T, 0}}
$$

where $W_{P E T, 0}$ and $W_{P E T, t}$ refer to weights of initial and at a specific reaction time, respectively. The selected operating conditions for alkaline hydrolytic experiments were: stirring rate $400 \mathrm{rpm}$, particle size $250 \mu \mathrm{m}$, and inert atmosphere $200 \mathrm{kPa} \mathrm{N}$.

For $10 \mathrm{~g}$ of PET flakes depolymerised in $150 \mathrm{ml}$ of a $6.7 \%$ aqueous sodium hydroxide solution $(\mathrm{NaOH}: \mathrm{PET}$ molar ratio $=5.76)$ at $60,70,80,90$ and $100{ }^{\circ} \mathrm{C}$, it was found that the amount of PET flakes depolymerised increased with temperature as a function of reaction time (fig. 1). Especially for the case at 100 ${ }^{\circ} \mathrm{C}$, most of the charged flakes $(>85 \%)$ were decomposed in four hours. After the removal of the remaining flakes by filtration and subsequent neutralisation of the liquid phase and filtration again, the liquid obtained was analysed by gas chromatography for organic products. Only ethylene glycol was found. On the other hand, results from titration revealed that high purity TPA was the major solid product obtained after neutralisation. DSC analysis of fresh and residual flakes suggested that remaining PET particles after reaction maintained their original structure, this suggesting that the process of depolymerisation in sodium hydroxide solution occurred on the external surface of the flakes, and these were lamellarly depolymerised [7]. This was consistent with the observed decrease in PET particle size with increasing conversion.

A simple theoretical model was developed to predict the time evolution of the reaction conversion. It should be pointed out that the derivation of a mathematical method is rather complicated since mass transfer phenomena between the solid and the liquid phase may occur in parallel to the chemical 


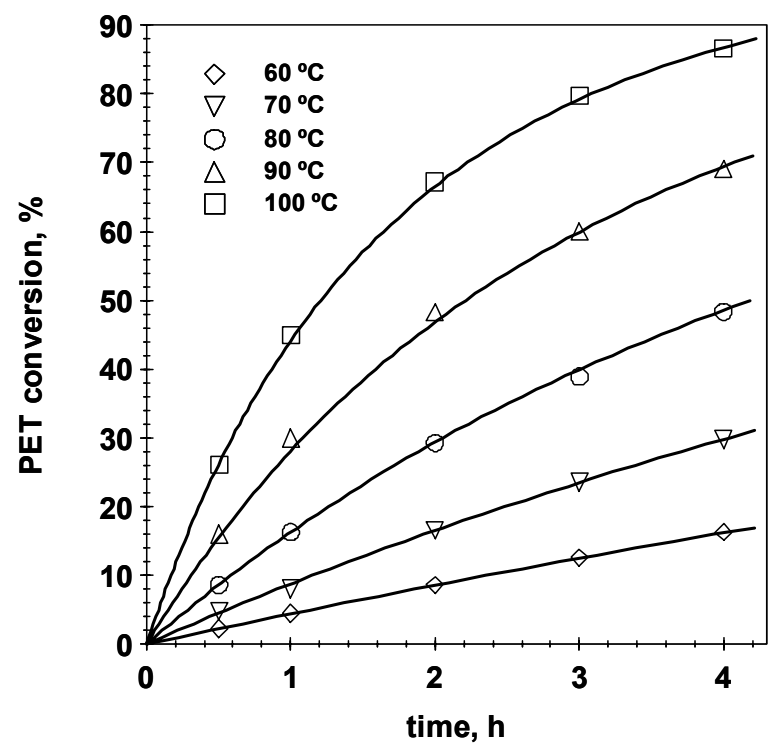

Figure 1: $\quad$ PET conversion in alkaline hydrolysis as a function of time at different temperatures $\left(60,70,80,90\right.$ and $\left.100{ }^{\circ} \mathrm{C}\right)$ with a $\mathrm{NaOH}: \mathrm{PET}$ molar ratio of 5.76. Solid lines represent theoretical modelling results.

reaction. Thus, a preliminary study on the effect of particle size $(170-510 \mu \mathrm{m})$ and stirring rate $(0-1000 \mathrm{rpm})$ on PET conversion was carried out. As a result, $250 \mu \mathrm{m}$ particle size and $400 \mathrm{rpm}$ were the values of these parameters that minimised the presence of external mass gradients. The reaction rate $\left(-\mathrm{r}_{\mathrm{A}}\right.$ in mol $\mathrm{l}^{-1} \mathrm{~h}^{-1}$ ) of alkaline hydrolysis of PET (or the production either of ethylene glycol or of disodium terephthalate) was defined by the molar consumption of PET per unit reaction volume. Since the product of TPA produced in the reaction was dissolved in the solution and became of its salt form, the terephthalic salt would be inactive in an eventual nucleophilic substitution for the esterification (the reverse reaction for the alkaline hydrolysis) [8]. Therefore, the reaction of PET hydrolysis under alkaline conditions could be considered as an irreversible reaction. The reaction rate equation could be simply expressed as the following power form:

$$
-r_{A}=k C_{P E T}^{a} C_{\mathrm{NaOH}}^{b}
$$

where $a$ and $b$ are the reaction orders with respect to PET and sodium hydroxide, respectively, and $k$ refers to the reaction rate constant. As an initial supposition, the reaction rate was considered to be proportional to the ester and alkali concentration $(a=b=1)$. If a constant volume for the reactions is assumed (150 $\mathrm{ml}$ ), the balance equation in terms of PET conversion $(X)$ and $\mathrm{NaOH}$ :PET molar ratio $(M)$ can be expressed as:

$$
\frac{1}{2-M} \ln \left(\frac{M(1-X)}{M-2 X}\right)=k C_{P E T, 0} t
$$


516 Waste Management and the Environment IV

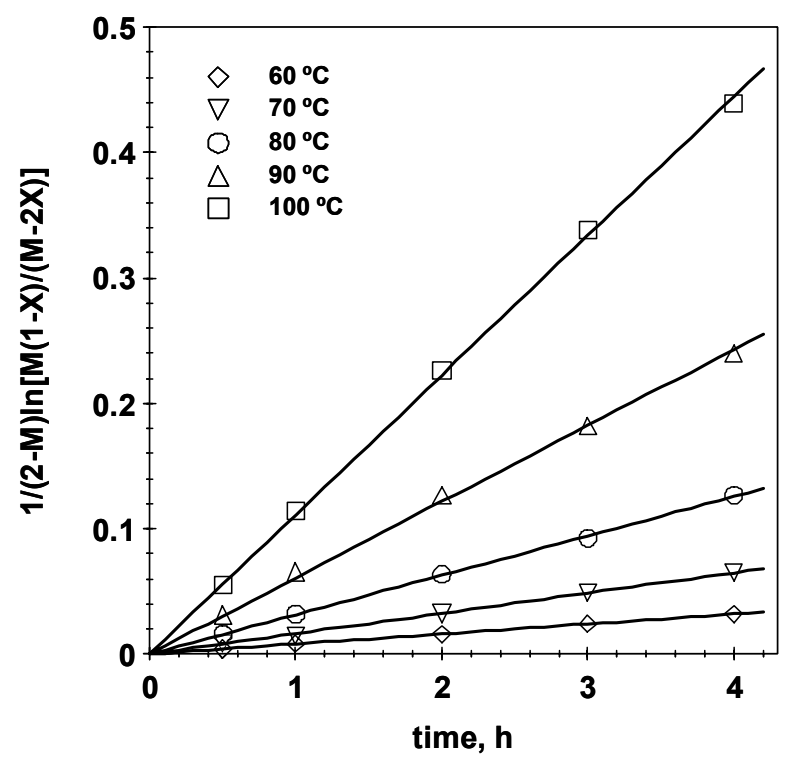

Figure 2: Fitting of kinetic data according to eqn. (4) at different temperatures $\left(60,70,80,90\right.$ and $\left.100^{\circ} \mathrm{C}\right)$ with a $\mathrm{NaOH}: \mathrm{PET}$ molar ratio of 5.76 .

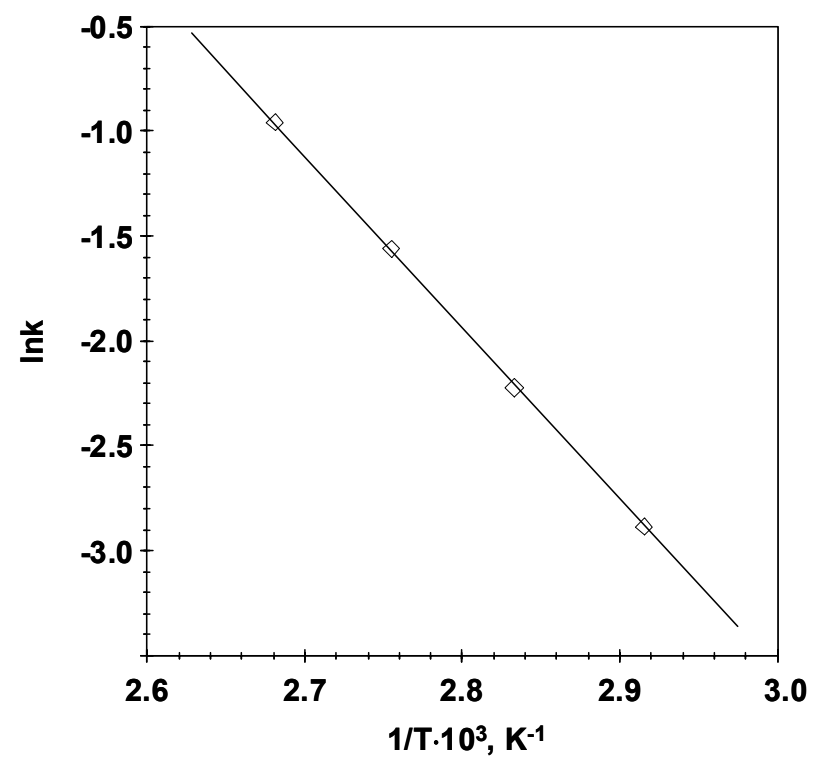

Figure 3: Arrhenius plot for the alkaline hydrolysis of PET with a $\mathrm{NaOH}:$ PET molar ratio of 5.76 . 
This kinetic model was examined by fitting the experimental data recorded at temperatures ranging between $60-100{ }^{\circ} \mathrm{C}$ at intervals of $10^{\circ} \mathrm{C}$. The results are shown in fig. 2 and indicated that this simple model provided a good linear relationships with linear correlation factors $\left(\mathrm{r}^{2}\right)$ higher than 0.99 in all cases. It could be therefore assumed that the reaction rate was first order to the PET concentration and first order to the $\mathrm{NaOH}$ concentration. The rate constants could be estimated from the slopes in the plot (fig. 2). As can be observed in fig. 1 the theoretical prediction values are in good agreement with the experimental data. According to the relationship of the rate constant with the reaction temperature, the Arrhenius plot is shown in fig. 3. The activation energy for the alkaline hydrolysis of PET calculated from the slope was $68 \pm 2 \mathrm{~kJ} \mathrm{~mol}^{-1}$. This value is in fairly good agreement with $69 \mathrm{~kJ} \mathrm{~mol}^{-1}$ reported by Wan et al. [9] for the alkaline depolymerisation with potassium hydroxide. Further, this activation energy value was quite lower than the corresponding value observed for acid hydrolysis, $100-110 \mathrm{~kJ} \mathrm{~mol}^{-1}[10,11]$. The pre-exponential factor calculated from the intercept was $1.3 \pm 0.7 \cdot 10^{9} 1 \mathrm{~mol}^{-1} \mathrm{~h}^{-1}$.

\subsection{Alkaline hydrolysis of PET assisted by phase transfer catalysts}

Phase transfer catalysts find applications in a variety of reactions, mainly related to the synthesis of organic and fine chemicals. Essentially the principle of PTC is based on the ability of certain 'phase-transfer agents (catalyst)' to facilitate the transport of one reagent from one phase into another (immiscible) phase wherein the other reagent exists. Within the context of alkaline hydrolysis of PET a potentially active PTC should efficiently transport the hydroxide anion from the aqueous phase to the organic phase (solid PET), thereby increasing the reaction rate. Recent studies have only evaluated the role of quaternary ammonium salts

[ $\left.{ }^{1} 2-14\right]$; however, no attention has been paid to examining the behaviour of tetralkyl phosphonium salts as PTCs (table 1) for this specific reaction system. For this screening study the following operating conditions were used: stirring rate $400 \mathrm{rpm}$, particle size $250 \mu \mathrm{m}$, inert atmosphere $200 \mathrm{kPa} \mathrm{N} \mathrm{N}_{2}$, temperature $80^{\circ} \mathrm{C}, \mathrm{NaOH}$ concentration $1.67 \mathrm{~mol} \mathrm{l}^{-1}$, PET concentration $0.29 \mathrm{~mol} \mathrm{l}^{-1}$, and PTC concentration $0.07 \mathrm{~mol} \mathrm{l}^{-1}$. The mixture was allowed to react for $0.5,0.75,1$ and 1.5 hours.

The activity results of the PTCs investigated are shown in table 2. It is clearly observed that six (4BuAB, 4BuACl, 4MPB, 4BuPB, 4BuPCl, and 4BuPH) out of nine PTCs resulted totally inactive in the reaction. In other words, conversion values were virtually identical to those recorded in the absence of PTC. Interestingly the remaining two salts outperformed all other catalysts. Thus, it was noticed that $3 \mathrm{Bu} 6 \mathrm{DPB}$ and $4 \mathrm{OPB}$ at $80{ }^{\circ} \mathrm{C}$ attained PET conversion values of 84 and $75 \%$, respectively, in one hour while the non-catalysed reaction only showed $16 \%$ conversion.

The remarkable reactivity of these two quaternary salts was assigned to a high compatibility with the organic phase and efficient anion transfer due to the highly lipophilic cation. Hence, the sufficient organic structure (large alkyl groups) was responsible for a substantial partition of the cation-anion pair into the organic phase [15]. In sum, these two PTCs fulfilled the requirements of 
having enough organic character in order to be lipophilic while small enough in order to avoid sterically hindering the reaction (to a larger extent for 4OPB compared with 3Bu6DPB). In contrast the lack of activity exhibited by $4 \mathrm{BuAB}$, $4 \mathrm{BuACl}, 4 \mathrm{MPB}, 4 \mathrm{BuPB}, 4 \mathrm{BuPCl}$, and $4 \mathrm{BuPH}$ was associated with their high solubility in water. Further, in an attempt to analyse the influence of the chemical nature of the central cation in the quaternary salt on the catalytic performance the activity of 4OPB was compared with that of 4OAB (tetraoctylammonium bromide). PET conversion results revealed that no significant differences were evident as conversion with $4 \mathrm{OAB}(73 \%)$ was quite similar to that observed for 4OPB (75\%), and that the PTC activity was thus mainly governed by the chemical nature of the alkyl groups in the quaternary salts.

Table 2: $\quad$ PET conversion (\%) with different PTCs at $80^{\circ} \mathrm{C}$.

\begin{tabular}{|c|c|c|}
\hline PTC & Reaction time: 1 hour & Reaction time: 1.5 hours \\
\hline No catalyst & 16.3 & 23.2 \\
\hline 4BuAB & 16.3 & 23.2 \\
\hline 4BuACl & 16.3 & 23.2 \\
\hline $4 \mathrm{BuPB}$ & 16.3 & 23.2 \\
\hline 4BuPCl & 16.3 & 23.2 \\
\hline 4BuPH & 16.3 & 23.2 \\
\hline 4MPB & 16.3 & 23.2 \\
\hline 4OAB & 74.2 & 83.0 \\
\hline 4OPB & 75.2 & 83.1 \\
\hline 3Bu6DPB & 84.1 & 95.9 \\
\hline
\end{tabular}

It is hypothesised that the cationic part of the catalyst (alkyl groups) carries the hydroxide anion into the surface of the organic phase by means an interfacial mechanism. In this way the PET macromolecules in the surface of the flakes can be easily attacked by the $\mathrm{OH}^{-}$and subsequently depolymerised. The terephthalate anion produced returns into the aqueous phase and forms the disodium terephthalate salt with the $\mathrm{Na}^{+}$. The reaction proceeds until complete depolymerisation of PET to $\mathrm{Na}_{2}$-TPA and ethylene glycol, while the catalyst remains in the aqueous phase.

Table 3: PET conversion (\%) with different PTC (3Bu6DPB) concentrations at several temperatures $\left(60,70\right.$ and $\left.80^{\circ} \mathrm{C}\right)$.

\begin{tabular}{|c|l|l|l|l|l|l|l|l|l|}
\hline $\mathrm{t}(\mathrm{h})$ & \multicolumn{2}{|c|}{$\mathrm{C}_{\text {PTC: }}: 0.02 \mathrm{~mol} \mathrm{l}^{-1}$} & \multicolumn{3}{c|}{$\mathrm{C}_{\text {PTC }}: 0.04 \mathrm{~mol} \mathrm{l}^{-1}$} & \multicolumn{3}{c|}{$\mathrm{C}_{\text {PTC: }}: 0.07 \mathrm{~mol} \mathrm{l}^{-1}$} \\
\hline & 60 & 70 & 80 & 60 & 70 & 80 & 60 & 70 & 80 \\
& $\begin{array}{l}6 \\
{ }^{\circ}\end{array}$ & ${ }^{\circ} \mathrm{C}$ & ${ }^{\circ} \mathrm{C}$ & ${ }^{\circ} \mathrm{C}$ & ${ }^{\circ} \mathrm{C}$ & ${ }^{\circ} \mathrm{C}$ & ${ }^{\circ} \mathrm{C}$ & $\begin{array}{l}{ }^{\circ} \mathrm{C} \\
{ }^{\circ} \mathrm{C}\end{array}$ \\
\hline 0.5 & 12.4 & 13.1 & 28.6 & 22.7 & 24.5 & 41.0 & 35.0 & 40.3 & 59.8 \\
\hline 0.75 & 18.4 & 21.1 & 40.6 & 28.3 & 32.0 & 53.4 & 40.0 & 52.8 & 73.6 \\
\hline 1 & 23.2 & 26.7 & 49.2 & 32.2 & 41.0 & 61.6 & 42.8 & 62.3 & 84.1 \\
\hline 1.5 & 27.3 & 40.0 & 60.3 & 37.3 & 52.0 & 88.3 & 53.4 & 79.1 & 95.9 \\
\hline
\end{tabular}


Table 3 summarises the influence of PTC (3Bu6DPB) concentration (0.02$\left.0.07 \mathrm{~mol} \mathrm{l}^{-1}\right)$ and reaction temperature $\left(60-80{ }^{\circ} \mathrm{C}\right)$ on PET conversion. As expected conversion was promoted with increasing catalyst concentration and temperature. At $80{ }^{\circ} \mathrm{C}$ a conversion about $90 \%$ conversion could be attained only after 1.5 hours with a PTC concentration as low as $0.04 \mathrm{~mol} \mathrm{l}^{-1}\left(\mathrm{C}_{\mathrm{PTC}}\right.$ : $\mathrm{C}_{\mathrm{PET}}=$ 0.125 ). Note that only $23 \%$ conversion was reached for the non-catalysed reaction. Further, the reaction rate was found to be proportional to the catalyst concentration.

\section{Conclusions}

Solid PET depolymerised in sodium hydroxide solution produced almost only terephthalic salt and ethylene glycol in the solution. A kinetic model considering that the reaction rate was first order with respect to both PET and $\mathrm{NaOH}$ concentrations adequately described the kinetics of the depolymerisation under alkaline conditions. The activation energy of the process was $68 \mathrm{~kJ} \mathrm{~mol}^{-1}$. Tributylhexadecylphosphonium bromide (3Bu6DPB) was discovered to be an active phase transfer catalyst for the process since it considerably accelerated the reaction rate. It is thought that this quaternary phosphonium salt act as a shuttling agent by extracting the $\mathrm{OH}^{-}$anion from the aqueous phase into the organic interfacial region (the surface of solid PET flakes) where the anion can freely react with the organic reactant. Thus, noticeable conversion of PET with considerably low catalyst concentration and energy inputs to the system (relatively low temperature ranging $70-80^{\circ} \mathrm{C}$ ) could be attained.

\section{Acknowledgements}

The financial support for this work provided the Spanish Ministerio de Educación y Ciencia (CTQ2005-06247) and Gobierno Vasco (FuturesEtortek 2007) is gratefully acknowledged.

\section{References}

[1] PETCORE, www.petcore.org

[2] Awaja, F. \& Pavel, D., Recycling of PET. European Polymer Journal 41(7), pp. 1453-1477, 2005.

[3] Paszun, D. \& Spychaj, T., Chemical recycling of poly(ethylene terephthalate). Industrial \& Engineering Chemistry Research, 36(4), pp. 1373-1383, 1987

[4] Karayannidis, G.P. \& Achilias, D.S., Chemical recycling of poly(ethylene terephthalate). Macromolecular Materials and Engineering, 292(2), pp. 128-146, 2007.

[5] Lorenzetti, C., Manaresi, P., Berti, C. \& Barbiroli, G., Chemical recovery of useful chemicals from polyester (PET) waste for resource conservation: a survey of state of the art. Journal of Polymers and the Environment, 14(1), pp. 89-101, 2006. 
[6] Campanelli, J.R., Cooper, D.G. \& Kamal, M.R., Catalyzed hydrolysis of poly(ethylene terephthalate) melts. Journal of Applied Polymer Science, 53(8), pp. 985-991, 1994.

[7] Kumar, S. \& Guria, C., Alkaline hydrolysis of waste poly(ethylene terephthalate): a modified shrinking core model. Journal of Macromolecular Science, Pure and Applied Chemistry, A42(3), pp. 237251, 2005.

[8] Mishra, S. \& Goje, A.S., Chemical recycling, kinetics, and thermodynamics of alkaline depolymerisation of waste poly(ethylene terephthalate) (PET). Polymer Reaction Engineering, 11(4), pp. 963-987, 2003.

[9] Wan, B-Z., Kao, C-Y. \& Cheng, W-H., Kinetics of depolymerization of poly(ethylene terephthalate) in a potassium hydroxide solution. Industrial \& Engineering Chemistry Research, 40(2), pp. 509-514, 2001.

[10] Yoshioka, T., Okayama, N. \& Okuwaki, A., Kinetics of hydrolysis of PET powder in nitric acid by a modified shrinking-core model. Industrial \& Engineering Chemistry Research, 37(2), pp. 336-340, 1998.

[11] Mancini, S.D. \& Zanin, M., Post consumer PET depolymerisation by acid hydrolysis. Polymer-Plastics Technology and Engineering, 46(2), pp. 135144, 2007.

[12] Polk, M.B., Leboeuf, L.L., Shah, M., Won, C-Y., Hu, X. \& Ding, W., Nylon 66, nylon 46, and PET phase-transfer-catalyzed alkaline depolymerization at atmospheric pressure. Polymer-Plastics Technology and Engineering, 38(3), pp. 459-470, 1999.

[13] Kosmidis, V.A., Achilias, D.S. \& Karayannidis, G.P., Poly(ethylene terephthalate) recycling and recovery of pure terephthalic acid. Kinetics of a phase transfer catalyzed alkaline hydrolysis. Macromolecular Materials and Engineering, 286(10), pp. 640-647, 2001.

[14] Das, J., Halgeri, A.B., Sahu, V. \& Parikh, P.A., Alkaline hydrolysis of poly(ethylene terephthalate) in presence of a phase transfer catalyst. Indian Journal of Chemical Technology, 14(2), pp. 173-177, 2007.

[15] Naik, S.D. \& Doraiswamy, L.K., Phase transfer catalysis: chemistry and engineering. AIChE Journal, 44(3), pp. 612-646, 1998. 